hep-ph/9711397

ANL-HEP-CP-97-96

November 1997

\title{
Photon Scattering in Muon Collisions ${ }^{1}$
}

\author{
Michael Klasen ${ }^{2}$ \\ Argonne National Laboratory ${ }^{3}$ \\ High Energy Physics Division \\ Argonne, Illinois 60439
}

\begin{abstract}
We estimate the benefit of muon colliders for photon physics. We calculate the rate at which photons are emitted from muon beams in different production mechanisms. Bremsstrahlung is reduced, beamstrahlung disappears, and laser backscattering suffers from a bad conversion of the incoming to the outgoing photon beam in addition to requiring very short wavelengths. As a consequence, the cross sections for jet photoproduction in $\mu p$ and $\mu^{+} \mu^{-}$collisions are reduced by factors of 2.2 and 5 compared to $e p$ and $e^{+} e^{-}$machines. However, the cross sections remain sizable and measurable giving access to the photon and proton parton densities down to $x$ values of $10^{-3}$ to $10^{-4}$.
\end{abstract}

\section{INTRODUCTION}

Muon colliders offer an interesting alternative and complement to future $e^{+} e^{-}$linear colliders. If the considerable design difficulties, in particular in the multistage cooling, the neutrino radiation problems arising from decaying muons, and the management of detector backgrounds, can be solved, they can be precisely tuned to a Higgs or $t \bar{t}$ factory, be ramped in several stages to much higher energies up to 3 or $4 \mathrm{TeV}$, and, due to a smaller total size, eventually be built at lower cost. In addition, an existing high energy proton beam might be collided with one of the muon beams to give a high energy lepton-proton collider. The physics interest focuses on measurements of the Higgs and weak gauge boson properties, top quark physics, supersymmetry with and without R-parity violation, and other phenomena beyond the Standard Model [1].

Little attention has been paid in this context to photon initial states and QCD measurements. This is surprising given the many studies at present and future $e^{+} e^{-}$colliders, the success of HERA in determining the proton structure and hard QCD processes, and the significance of QCD background for many

1) Talk given at the Workshop on Physics at the First Muon Collider, Fermilab, Nov. 1997.

2) E-mail: klasen@hep.anl.gov.

3) Work supported by the U.S. Department of Energy, Division of High Energy Physics, Contracts W-31-109-ENG-38 and DEFG05-86-ER-40272. 
of the new physics processes under consideration. At LEP, ALEPH, DELPHI, and OPAL have studied the photon structure function $F_{2}^{\gamma}\left(x, Q^{2}\right)$ in electronphoton scattering [2], and L3 and OPAL have obtained first measurements of the energy dependence of the total $\gamma \gamma$ cross section for hadron production [3]. Whereas LEP1 was dominated by $e^{+} e^{-}$annihilation at the $Z$ pole, $\gamma \gamma$ scattering is important at LEP2 and will be dominant at a future linear $e^{+} e^{-}$ collider. This is due to the fact that the annihilation cross section drops like $\sigma_{l^{+} l^{-}} \propto \frac{1}{s}$ or at best like $\frac{\log s}{s}(l=e, \mu)$, whereas the photon-photon cross section rises like $\sigma_{\gamma \gamma} \propto \log ^{3} s$ or even $\propto s$ taking into account the hadronic structure of the photon. This leads us naturally to the presumption that photoproduction might not be as negligible in muon collisions as is widely believed.

Literature on muon colliders in general and on photon radiation by muons in particular is very restricted if not absent. However, two detailed studies on two-photon physics at $e^{+} e^{-}$linear colliders exist $[4,5]$, where the authors discuss photon emission and distribution functions, soft and hard two-photon reactions, and total cross sections. This study follows the lines given in these references, looks for changes from $e^{+} e^{-}$to $\mu^{+} \mu^{-}$, extends them to $\mu p$ collisions and compares these to $e p$ collisions. In the following section, we estimate the production rate of photons from muon beams in three different mechanisms. The results are used in the third section to calculate the cross section for dijet production as a function of the transverse energy of the jets and the center-ofmass energy of the muon pair. Finally, we determine the ranges in $x$ in which the photon and proton structure functions could be measured at a $\mu^{+} \mu^{-}$and $\mu p$ collider.

\section{(QUASI-)REAL PHOTON EMISSION}

Three mechanisms can contribute to the emission of photons from leptons: bremsstrahlung, beamstrahlung, and laser backscattering. To begin with, bremsstrahlung is an approximation of the complete two-photon process $l^{+} l^{-} \rightarrow l^{+} l^{-} X$, where $l$ can be an electron or a muon, and $X$ is a hadronic final state in our case. The photons are radiated from the leptons in the scattering process, and their spectrum can be expressed through the usual Weizsäcker-Williams or Equivalent Photon Approximation [6]

$$
f_{\gamma / l}^{\text {brems }}(x)=\frac{\alpha}{2 \pi}\left[\frac{1+(1-x)^{2}}{x} \log \frac{Q_{\max }^{2}}{Q_{\min }^{2}}+2 m_{l}^{2} x\left(\frac{1}{Q_{\max }^{2}}-\frac{1}{Q_{\min }^{2}}\right)\right],
$$

where $x=\frac{E_{\gamma}}{E}$ is the fraction of the photon energy $E_{\gamma}$ of the lepton beam energy $E$, and where the virtuality of the photon

$$
Q^{2}=\frac{m_{l}^{2} x^{2}}{1-x}+E^{2}(1-x) \theta^{2}+\mathcal{O}\left(E^{2} \theta^{2}, m_{l}^{2} \theta^{2}, \frac{m_{l}^{2}}{E^{2}}\right)
$$


is always smaller than

$$
Q^{2}=-\left(p_{l}-p_{l^{\prime}}\right)^{2}=2 E E^{\prime}(1-\cos \theta)<4 E E^{\prime}=4 E^{2}(1-x) .
$$

This upper limit on $Q^{2}$ is used if no information on the scattered lepton is available. For a safe factorization of the lepton tensor and phase space, it is preferred to anti-tag the outgoing lepton and use the maximum scattering angle $\theta$ in Eq. (2). Consequently, the photon density will depend on this maximum scattering angle. This is shown in Table 1 for a $\sqrt{s}=500 \mathrm{GeV}$ $e^{+} e^{-}$collider. The variation in this case can reach up to $40 \%$. Which changes

TABLE 1. Dependence of the photon density on the maximum scattering angle.

\begin{tabular}{|c|c|}
\hline$\theta_{\max }$ & $f_{\gamma / e}^{\text {brems }}(x=0.5)$ \\
\hline \hline- & 0.078 \\
$10 \mathrm{mrad}$ & 0.047 \\
$20 \mathrm{mrad}$ & 0.051 \\
$30 \mathrm{mrad}$ & 0.053 \\
\hline
\end{tabular}

occur in the Weizsäcker-Williams spectrum for muon beams? Eq. (1) depends explicitly on the lepton mass $m_{l}$ in its non-logarithmic contribution as well as implicitly through the minimal photon virtuality $Q_{\min }^{2}=\frac{m_{1}^{2} x^{2}}{1-x}$. Taking into account only the leading logarithmic contribution, we expect a reduction by a factor of

$$
\frac{\log \frac{Q_{\max }^{2}(1-x)}{m_{\mu}^{2} x^{2}}}{\log \frac{Q_{\max }^{2}(1-x)}{m_{e}^{2} x^{2}}}=\frac{-\log m_{\mu}^{2}}{-\log m_{e}^{2}} \simeq \frac{1}{3.38},
$$

where we have taken $Q_{\max }^{2}=1 \mathrm{GeV}^{2}$ and $1-x=x^{2}$ or $x=0.618$ for simplicity. If one considers a muon collider with a photon spectrum on either side, one expects the cross section to be reduced by a factor of $\left(\frac{1}{3.38}\right)^{2} \simeq \frac{1}{11.4}$. In Figure 1, we compare the Weizsäcker-Williams (WW) spectra of electrons and muons as a function of $x$. We have not assumed any anti-tagging conditions on the scattered lepton. The shape of the muon spectrum is basically unchanged with respect to the electron spectrum, and the normalization drops by a factor of two. This is in qualitative agreement with our naïve estimate above.

A second contribution to photon emission comes from beamstrahlung. The particles in one bunch experience rapid acceleration when they enter the electromagnetic field of the opposite bunch. Then the scattering amplitudes between particles within the characteristic length add coherently. This can involve up to $10^{6}$ particles. The intensity and spectrum of the beamstrahlung depend sensitively on the size and shape of the bunches and thus on the machine parameters. It is known that for particular $e^{+} e^{-}$linear collider designs 


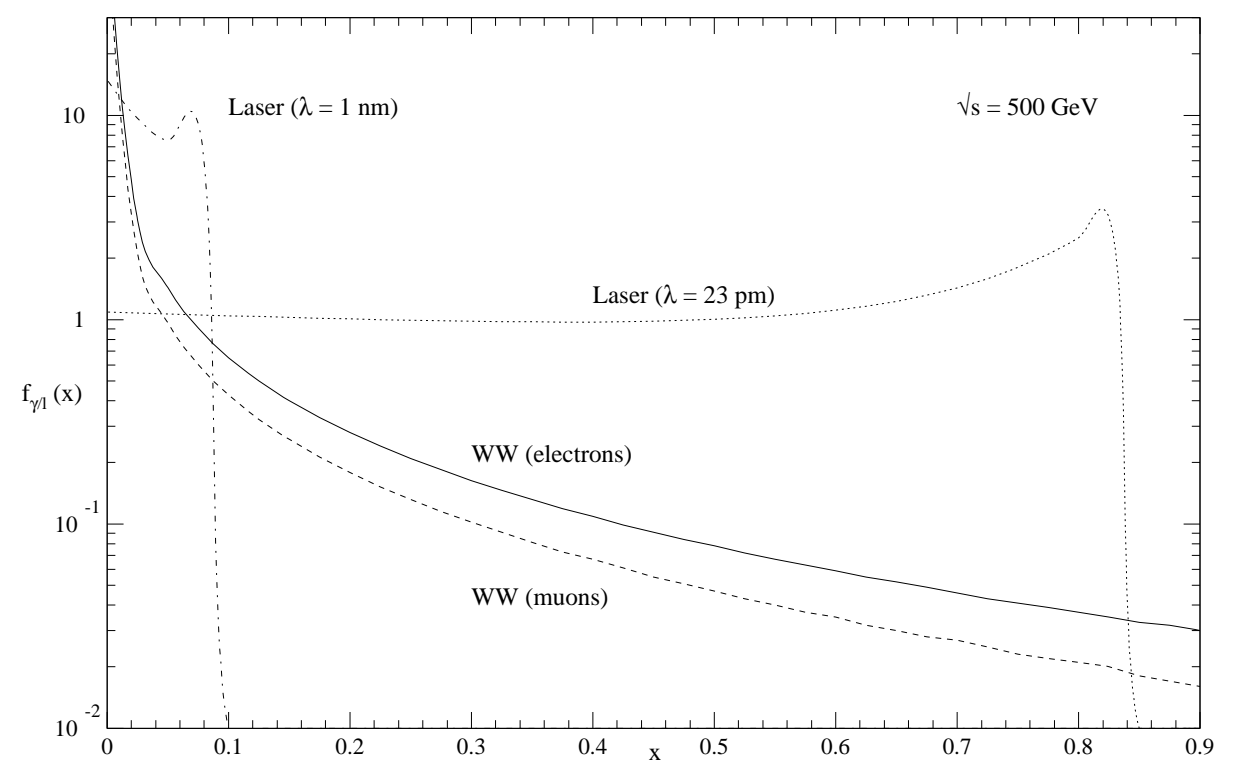

FIGURE 1. (Quasi-)real photon emission at a $\sqrt{s}=500 \mathrm{GeV} \mu^{+} \mu^{-}$collider. We show the Weizsäcker-Williams (WW) spectra for electrons and muons and the spectra for laser backscattering off muons for incident photons with wavelengths of $23 \mathrm{pm}$ and $1 \mathrm{~nm}$.

beamstrahlung can be more important than bremsstrahlung over a wide range in $x$ [4]. Within a semiclassical calculation and for a Gaussian longitudinal bunch profile, Chen et al. find

$$
f_{\gamma / l}^{\text {beam }}(x)=\frac{1}{\Gamma\left(\frac{1}{3}\right)}\left[\frac{2}{3 \mathcal{Y}}\right]^{1 / 3} x^{-2 / 3}(1-x)^{-1 / 3} \exp \left[-\frac{2 x}{3 \mathcal{Y}(1-x)}\right] G(x),
$$

where the function $G(x)$ is of order 1 and can found in [5]. The beamstrahlung intensity is controlled by the effective beamstrahlung parameter

$$
\mathcal{Y}=\frac{5 r_{l}^{2} E N}{6 \alpha \sigma_{z}\left(\sigma_{x}+\sigma_{y}\right) m_{l}}
$$

mostly through the exponential function.

$$
r_{l}=\frac{e^{2}}{4 \pi \epsilon_{0} m_{l} c^{2}}
$$

is the "classical radius" of each of the $N$ leptons with mass $m_{l}$ in a bunch of length $\sigma_{z}$ and transverse dimensions $\sigma_{x}$ and $\sigma_{y}$. $E$ is the beam energy, and $\alpha=\frac{e^{2}}{4 \pi}$ is the fine structure constant. The beamstrahlung parameter $\mathcal{Y}$ depends explicitly and implicitly through the classical lepton radius on the 
lepton mass $m_{l}$. We therefore expect the beamstrahlung parameter for muon beams to be reduced by

$$
\frac{\mathcal{Y}_{\mu}}{\mathcal{Y}_{e}}=\frac{m_{e}^{3}}{m_{\mu}^{3}} \simeq 8 * 10^{-6}
$$

i.e. by three powers of the mass ratio of electrons to muons. Since the beamstrahlung intensity will be suppressed by this factor exponentially, we do not expect any beamstrahlung for muon beams and in fact do not see any in a numerical simulation using the formula of Eq. (5).

$e^{+} e^{-}$colliders can also be run in a mode dedicated to $\gamma \gamma$ collisions using backscattering of laser light off the incident electron and positron beams. One considers even dedicated interaction regions. For unpolarized lepton beams, the spectrum of the backscattered photons depends only on the beam energy, on the laser wavelength, and - this is crucial for muon beams - on the beam particle mass. Ginzburg et al. determined this spectrum to be [7]

$$
f_{\gamma / l}^{\text {laser }}(x)=\frac{1}{N}\left[1-x+\frac{1}{1-x}-\frac{4 x}{X(1-x)}+\frac{4 x^{2}}{X^{2}(1-x)^{2}}\right],
$$

where the normalization is

$$
N=\left[1-\frac{4}{X}-\frac{8}{X^{2}}\right] \log (1+X)+\frac{1}{2}+\frac{8}{X}-\frac{1}{2(1+X)^{2}}
$$

As already stated above, the crucial parameter

$$
X=\frac{4 E E_{\gamma}^{\text {in }}}{m_{l}^{2}}
$$

in this case depends on the beam energy $E$, on the incident photon energy $E_{\gamma}^{\text {in }}=\frac{h c}{\lambda}$, and on the lepton mass $m_{l}$. Telnov estimates the optimal value of $X$ from the threshold of the $e^{+} e^{-}$pair creation in photon collisions being

$$
E_{\gamma}^{\text {out }} E_{\gamma}^{\text {in }}=\frac{X^{2} m_{l}^{2}}{4(X+1)}>m_{e}^{2}
$$

For electron beams, $m_{l}=m_{e}$ cancels in the equation above leading to an optimal value of $X_{e}^{\text {opt }}=2(1+\sqrt{2})$. For muon beams, we find

$$
X_{\mu}^{\mathrm{opt}}=2 \frac{m_{e}^{2}}{m_{\mu}^{2}}\left(1+\sqrt{1+\frac{m_{\mu}^{2}}{m_{e}^{2}}}\right) \simeq 9.72 * 10^{-3} .
$$

In Table 2 we calculate the optimal laser wavelength as a function of the center-of-mass energy $\sqrt{s}$ of the lepton collider or equivalently the lepton beam energy $E_{e, \mu}$. For electron beams, the optimal wavelengths lie in the region of 
TABLE 2. Dependence of the laser wavelength on the center-of-mass energy.

\begin{tabular}{|c|c|c|c|c|}
\hline$\sqrt{s} / \mathrm{GeV}$ & $E_{e, \mu} / \mathrm{GeV}$ & $\lambda_{\gamma}^{e} / \mathrm{nm}$ & $\lambda_{\gamma}^{\mu, \text { equiv }} / \mathrm{pm}$ & $\lambda_{\gamma}^{\mu, \text { opt }} / \mathrm{nm}$ \\
\hline \hline 100 & 50 & 197 & 4.6 & 2.29 \\
200 & 100 & 393 & 9.2 & 4.57 \\
350 & 175 & 688 & 16.1 & 8.00 \\
500 & 250 & 983 & 23.0 & 11.4 \\
\hline
\end{tabular}

visible light (197 - $983 \mathrm{~nm}$ ) and can be provided by current laser technology. To obtain the same backscattered photon spectrum as with electron beams, much shorter wavelengths are needed at a muon collider $(4.6-23.0 \mathrm{pm})$. The spectrum for $\lambda=23 \mathrm{pm}$ is shown in Figure 1. This spectrum does, however, not take into account the bad conversion of the incoming to the outgoing photon beam due to enhanced $e^{+} e^{-}$pair creation. If one chooses the optimal value of $X_{\mu}^{\mathrm{opt}}=9.72 * 10^{-3}$ for $100 \%$ conversion and no $e^{+} e^{-}$pair production, one finds incident laser wavelengths of 2.29 - $11.4 \mathrm{~nm}$. However, a laser beam of $11.4 \mathrm{~nm}$ produces backscattered photons at much too low energies which do not appear on the spectrum of Figure 1 at all. For illustration, we show the spectrum for $\lambda=1 \mathrm{~nm}$, where the spectrum is still concentrated at low energies, but at least visible. However, the conversion will be bad, and these short wavelengths can only be obtained with new laser technology like free electron lasers.

\section{QCD AND $\gamma \gamma$ SCATTERING}

In this section we will restrict ourselves to the reduced bremsstrahlung spectrum of muon beams and apply it to QCD and $\gamma \gamma$ scattering. As an example, we compare the differential dijet cross section $\mathrm{d} \sigma / \mathrm{d} E_{T}^{2-\text { jet }}$ at LEP2 and a muon collider of the same center-of-mass energy $\sqrt{s}=166.5 \mathrm{GeV}$ as a function of the transverse energy $E_{T}$ in Figure 2. This cross section has recently been measured by OPAL for $3 \mathrm{GeV}<E_{T}<20 \mathrm{GeV},-2<\eta_{1,2}<2$, and a maximum electron scattering angle of $\theta=33 \mathrm{mrad}$ using an integrated luminosity of about $20 \mathrm{pb}^{-1}$ [3]. It drops from 133 to $0.16 \mathrm{pb} / \mathrm{GeV}$ from the first to the last $E_{T}$-bin. The prediction for a muon collider is reduced by factors of 4.64 to 6.47 in this $E_{T}$-range. The cross section is still large enough to be measured with good precision.

It is also interesting to look at the dependence of the total dijet cross section on the center-of-mass energy. The result is shown in Figure 3 for two cuts on the transverse energy at 10 and $20 \mathrm{GeV}$. We observe a linear rise of the cross section as we expect when taking into account the hadronic structure of the photon. Photoproduction of jets will therefore increase linearly in importance with the energy at which a muon collider is operated. 


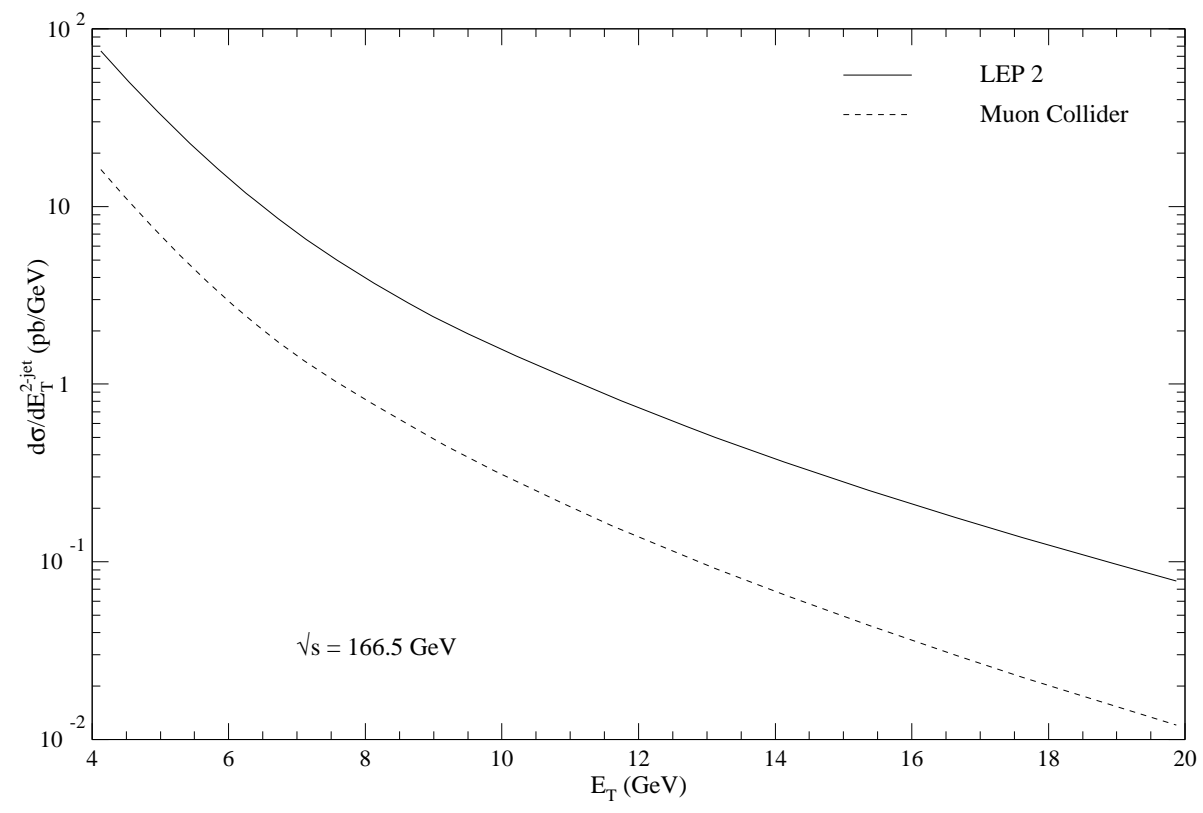

FIGURE 2. Comparison of the differential dijet cross section at LEP2 and a muon collider of the same center-of-mass energy as a function of the transverse energy $E_{T}$.

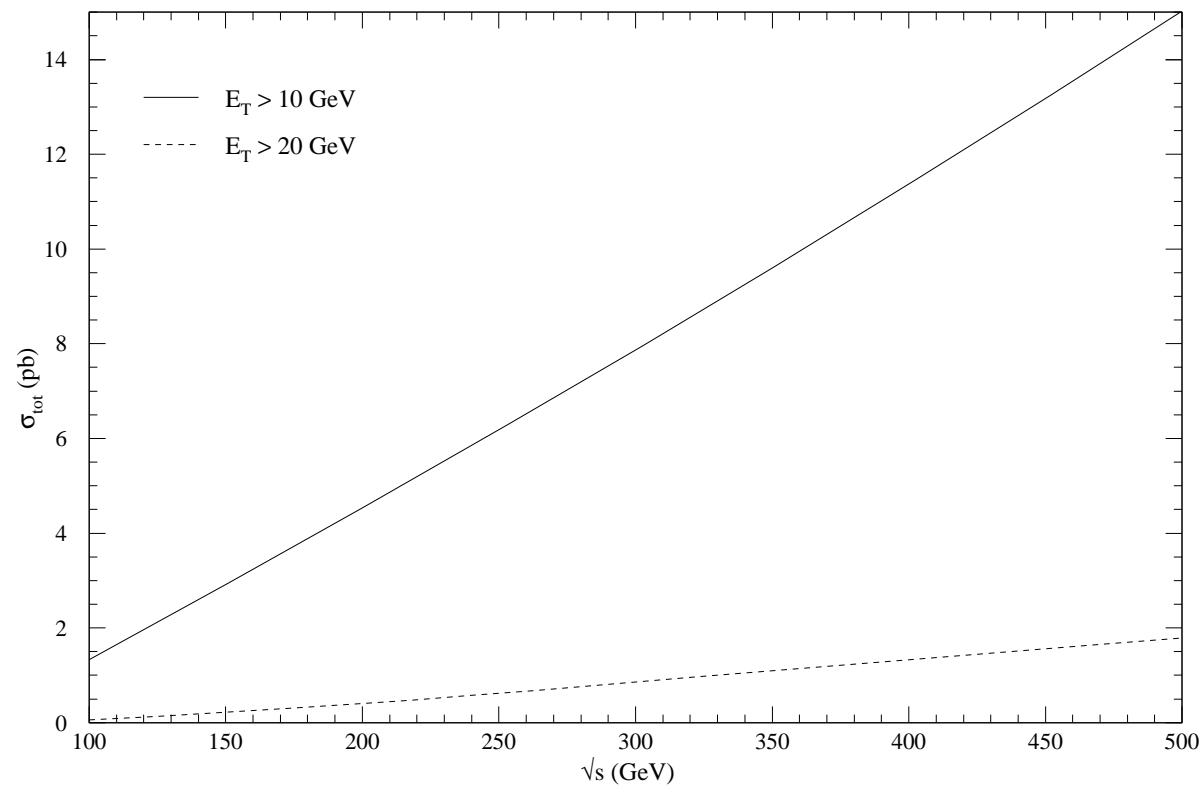

FIGURE 3. Center-of-mass energy dependence of the total dijet cross section for two thresholds of the transverse energy $E_{T}$. 


\section{PARTON DENSITY MEASUREMENTS}

We will now investigate the prospects for measuring the photon and proton parton densities at $\mu^{+} \mu^{-}$and $\mu p$ colliders in photoproduction processes. The $x$-ranges that are accessible can be calculated from four-momentum conservation and therefore depend completely on the kinematics of the initial and final states. The momentum fraction $x_{b}$ of a parton $b$ in the correspondent parent particle (muon or proton) is given by

$$
x_{b}=\frac{x_{a} E_{a} E_{T} e^{\eta_{1}}}{2 x_{a} E_{a} E_{b}-E_{b} E_{T} e^{-\eta_{1}}} .
$$

It depends on the momentum fraction $x_{a}$ of parton $a$ in its parent particle, on the beam energies $E_{a, b}$, on the transverse momentum of the outgoing particles $E_{T}$, and on the rapidity $\eta_{1}$ of one of these. The second rapidity $\eta_{2}$ is linearly dependent on these variables. We have plotted this function in Figure 4 for a muon collider with $\sqrt{s}=500 \mathrm{GeV}$ and different rapidities $\eta_{1} \in[-2 ; 2]$. This range corresponds to the fairly limited rapidity coverage of a detector at muon colliders due to massive shielding of decay electrons from the muon beams. The transverse energy $E_{T}=10 \mathrm{GeV}$ has been chosen in such a way that one may hope for a sufficient suppression of non-perturbative effects and a good determination of the energy scale which are indispensable for a safe extraction of the parton densities. Figure 4 also shows the envelope

$$
x_{b, \mathrm{env}}=\frac{E_{T}^{2}}{x_{a} E_{a} E_{b}},
$$

which gives the absolute limits on $x_{a, b}$ independent of the rapidity $\eta_{1}$. We find that for the kinematical conditions described above, rather low values of $x_{a, b}>1.6 * 10^{-3}$ can be reached where little is known about the parton densities (in particular of the gluon) in the photon.

Since it is not clear at which energy a muon collider will eventually operate, we also consider the center-of-mass energy dependence of the minimal $x$ value that can be reached in Figure 5. For $E_{T}=10$ (20) GeV, one can only go down to $0.04(0.16)$ for a $100 \mathrm{GeV}$ collider. Higher energies are therefore a clear advantage for photoproduction both for higher cross sections and for larger $x$-ranges.

Finally, we repeat the above analysis for a $200 \mathrm{GeV}$ x $1000 \mathrm{GeV}$ muon-proton collider. The result is shown in Figure 6. We find that the parton densities in the photon and proton could be measured down to values of $\mathcal{O}\left(5 * 10^{-4}\right)$.

\section{CONCLUSIONS}

We have studied several aspects of photon physics at muon colliders. Photon emission due to bremsstrahlung is found to be reduced, there is no beamstrahlung, and laser backscattering appears to be difficult. However, QCD 


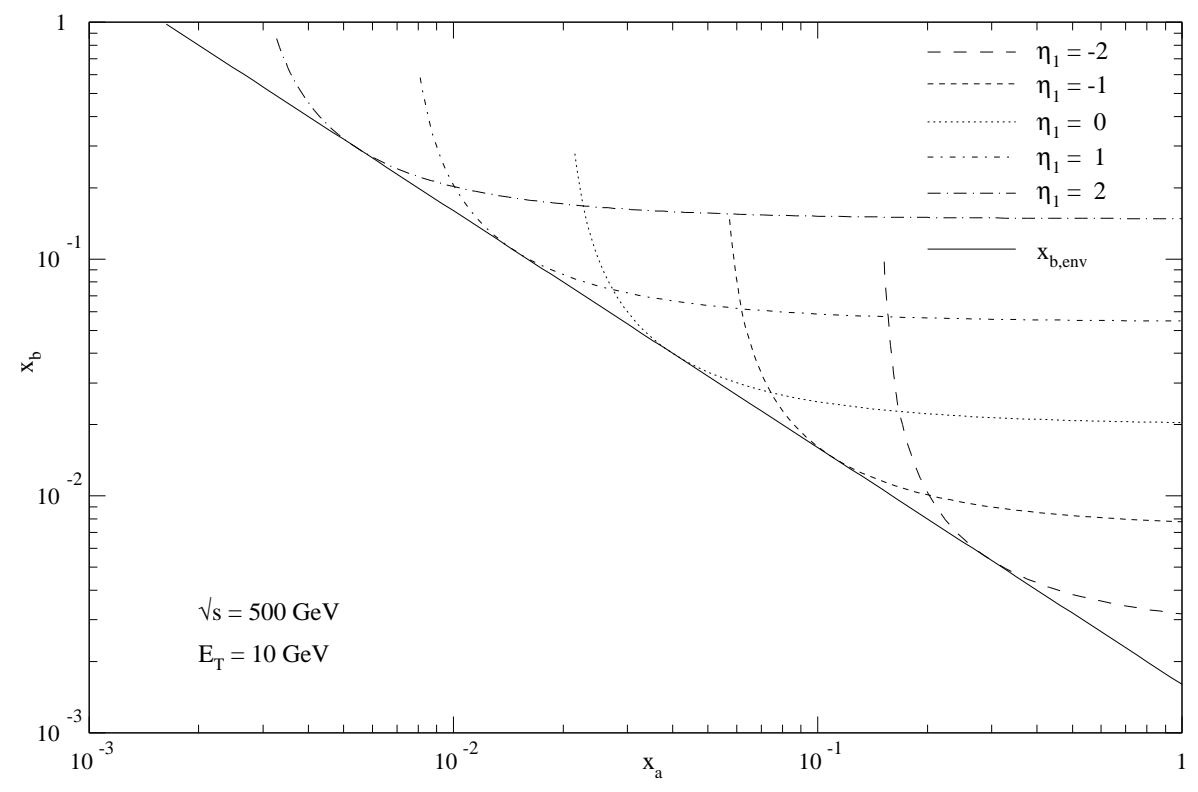

FIGURE 4. Parton density regions in the $x_{a}-x_{b}$ plane that can be accessed at $a \sqrt{s}=500$ $\mathrm{GeV}$ muon collider for different rapidities $\eta_{1}$ and $E_{T}=10 \mathrm{GeV}$.

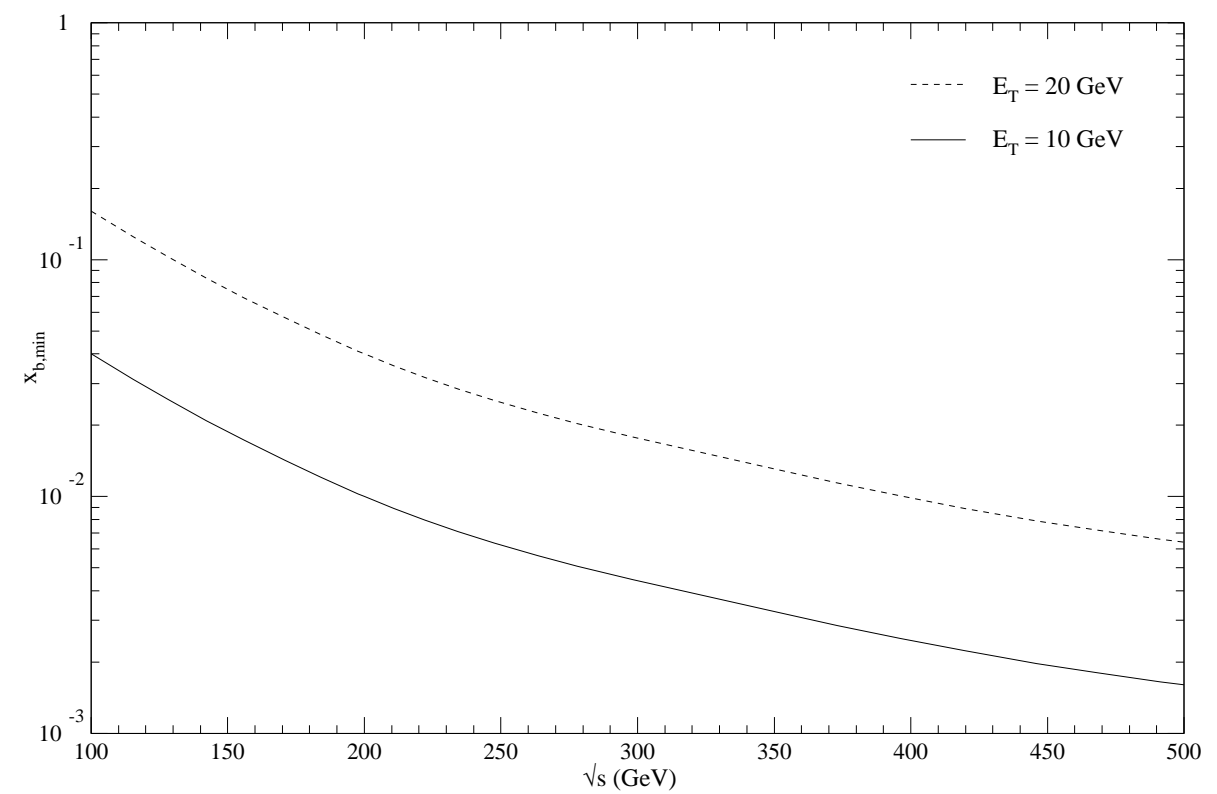

FIGURE 5. Center-of-mass energy dependence of the minimal $x_{b}$ value for two different transverse energies $E_{T}=10$ and $20 \mathrm{GeV}$. 


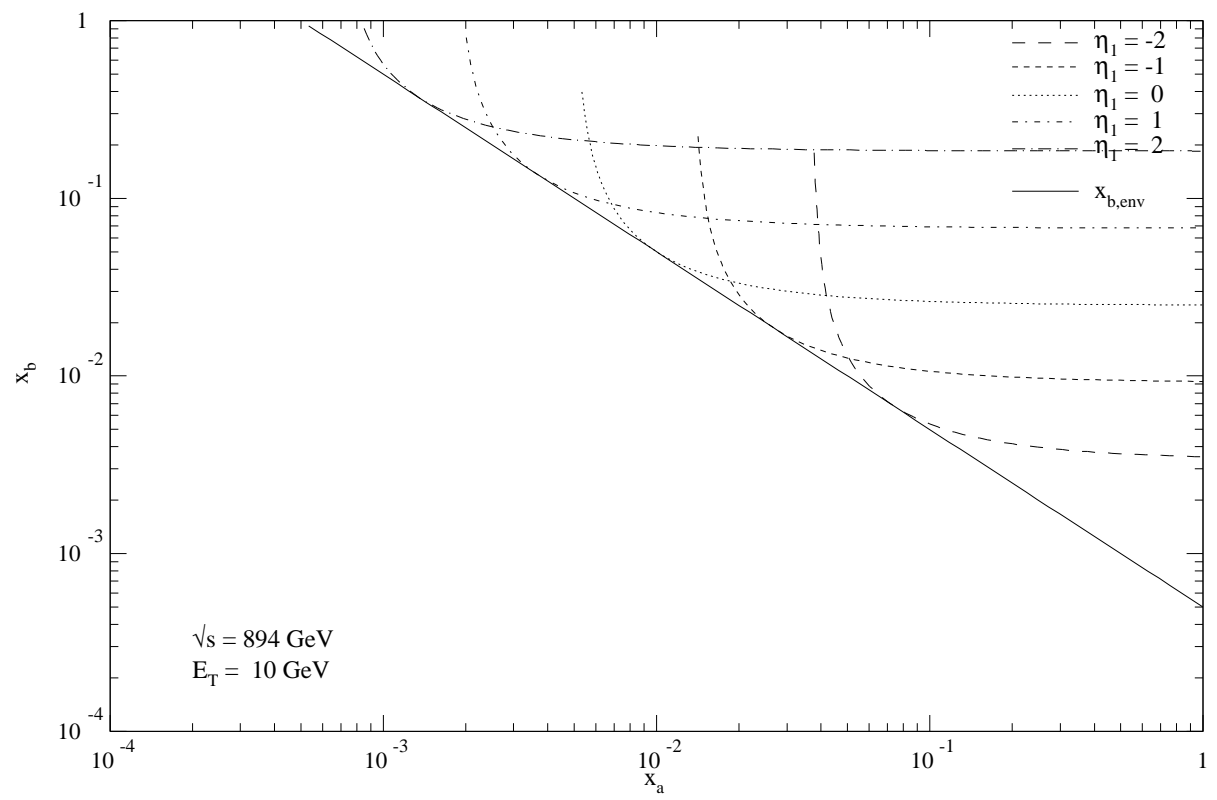

FIGURE 6. Parton density regions in the $x_{a}-x_{b}$ plane that can be accessed at $a \sqrt{s}=894$ $\mathrm{GeV}$ muon-proton collider for different rapidities $\eta_{1}$ and $E_{T}=10 \mathrm{GeV}$.

cross sections like photoproduction of dijets remain sizable and increase linearly with the center-of-mass energy. Photon and proton parton densities could be measured down to $x$ values of $10^{-3}$ to $10^{-4}$.

\section{REFERENCES}

1. Gunion J.F., in: Proc. of Beyond the Standard Model V, Balholm, Norway, May 1997, hep-ph/9707379.

2. ALEPH Coll., paper LP-315 submitted to LP 97, Hamburg, Germany; DELPHI Coll., Z. Phys. C69 (1996) 223; DELPHI Coll., paper 416 submitted to EPS 97, Jerusalem, Israel; OPAL Coll., Z. Phys. C74 (1997) 33; OPAL Coll., CERNPPE/97-87, hep-ex/9708019; OPAL Coll., CERN-PPE/97-103, hep-ex/9708028.

3. Söldner-Rembold S., plenary talk P01 at LP 97, Hamburg, Germany, hepex/9711005.

4. Drees M., and Godbole R.M., Z. Phys. C59 (1993) 591.

5. Chen P., Barklow T.L., and Peskin M.E., Phys. Rev. D49 (1994) 3209.

6. Frixione S., Mangano M.L., Nason P., and Ridolfi G., Phys. Lett. B319 (1993) 339.

7. Ginzburg I.F., Kotkin G.L., Serbo V.G., and Telnov V.I., Nucl. Instrum. Methods 205 (1983) 47; Ginzburg I.F., Kotkin G.L., Panfil S.L., Serbo V.G., and Telnov V.I., Nucl. Instrum. Methods 219 (1984) 5; Telnov V.I., Nucl. Instrum. Methods 294 (1990) 72. 